\title{
A Self-organizing Network of Alterable Competitive Layer for Pattern Cluster
}

\author{
Zhiling Wang, G. Sylos Labini and Marco De Sario ${ }^{\dagger}$ \\ Center for Space Geodesy, Italian Space Agency, P.O. Box 11, 75100 Matera, Italy \\ $\dagger$ Dept. of Electric Engineering-University of Bari, Via Re David,200-70125 Bari-Italy
}

\section{Introduction}

This paper apply ART1, only for binary input patterns, to perform the classification of image features. The category number of the competitive layer can be altered automatically to improve the net plasticity. There are two smoothing approaches used to reduce the effection of the noise on the extraction of image features. Fuzzy mapping functions are used to solve the un uncertainty/ambiquity problem of the image.

\section{System Architecture}

Layer 0: consist of a gray image. Its data can be used both to segment the image edges, textures etc. and to run the more complex segmentation algorithm when the learning fails.

Layer 1: include the neurons presenting different image features with binary $0 / 1$.

Layer 2: the alterable competitive layer to provide the cluster of image features.

\section{Feature Extraction}

Smoothing operation: Average of of pixels in $\mathrm{n}$ frame images is used when the camera is static; average of pixels in a window of a frame image is taken when it is moving.

Extraction of Feature: Here the multi-region fuzzy mapping function is used to estimate the distributions of the sizes, heights, textures, colour, ratios of wide/length, and adjacent/above/left relationships of regions in the image. The membership values are translated to binary form for the ART1 network,

\section{Neural Networks Training}

The algorithm for growthing neurons of competitive layer in improved ART1 is:

Step 1: Allocate arrays $w_{i j}^{\prime}, w_{j i}^{\prime}$, and $v_{i}^{\prime}$ as same dimensions as $w_{i j}, w_{j i}$, and $L_{2} v_{i}$.

Step 2: Copy all contents of the current arrays to the new medium arrays.

Step 3: Free the current arrays.

Step 4: The number of neurons in the $L_{2}$ is added by one, i.e. $\mathrm{M}+1$.

Step 5: Allocate arrays $w_{i j}, w_{j i}$, and $v_{i}$ again by the neuron number added $(\mathrm{M}+1)$.

Step 6: Copy the contents of the medium arrays to the first $\mathrm{M}$ items of $w_{i j}, w_{j i}$, and $v_{i}$.

Step 7: Initialize the weights of the $(\mathrm{M}+1)$ th neuron:

$$
w_{N+1 j}=\frac{1 .}{1 .+N} ; w_{j N+1}=1 \text {. }
$$

Step 8: Let the $(\mathrm{M}+1)$ th be a winning neuron and calculate its output.

Step 9: Free the medium arrays allocated in the step 1.

Step 10: Return to the improved ART1 algorithm to adjust weights of the winning neuron. Experiments

Here an $512 \times 512$ image with 255 gray-level grades is considered. In plasticity test, the neuron number of competitive layer is supposed as 4, arbitrarily. The set of 1127 frame images with binary is adapted. There are other tests having been done to compare the learning time of alterable neuron number of competitive layer with the fixed number. The correction of pattern classification by the improved algorithm is $100 \%$.

\section{Conclusion}

In this paper, two ways are used to smooth the image noise. The fuzzy mapping functions are applied to extract the image features. Because the category number of the competitive layer can be altered automatically by growing neuron number of the competitive layer, The network is able to work normally when the new patterns are inputed, i.e. good plasticity. The series of experiments have been completed. The all programs are written by $\mathrm{C}$ language and have been performed on a SUN-4/330 sparc-station system with an image board IT-150 and a CCCD camera. 